\title{
Overgrowth and Crystalline Structure of Gold Nanorods
}

\author{
J. B. Rodríguez-González, E. Carbó-Argibay, I. Pastoriza-Santos, J. Pérez-Juste and \\ L.M. Liz-Marzán
}

Department of Physical Chemistry, CSIC Associated unit-University of Vigo, 36310 Vigo, Spain

The effective control over the behavior of nanostructured systems requires a tight control over the shape and size of the nanoparticle building blocks. This control can be very effective and useful in the case of crystalline gold nanoparticles [1]. In order to achieve a precise morphology control over the particles obtained using gold nanorods as seeds; we have studied the crystalline structure of the initial single-crystal gold nanorods, which can be used in subsequent overgrowth processes. We also studied the mechanisms involved in the overgrowth and reshaping of such gold nanorods.

In the first part of this work we present TEM data on the structure of single-crystal gold Nanorods (Figure 1). The crystalline structure is interpreted on the basis of HRTEM analysis on the top view of standing nanorods, which shows clear evidence that the $\langle 100\rangle$ and $\langle 110\rangle$ crystallographic directions point toward lateral edges, and thus the faces joining on them must be assigned to higher indexes, which appear to be of $\{250\}$ type [2]. This is in contradiction with the assignment by Wang et al [3] and thus many of the interpretations regarding the growth, reactivity, and adsorption properties of gold nanorods must be revisited. Very recently, STEM tomography data has been published, which seems to confirm our statement of the need for a new crystalline model for single-crystal gold nanorods [4].

In the second part we show growth studies conducted on pentatwinned gold Nanorods that are made up of five twins with a common longitudinal twinning axis. This represents a fundamental point and drives the whole growth process, because the intermediate and final particles are also pentatwinned [5]. To follow the growth process and to determine the shape, the size and the crystallography of the intermediate and final particles we used transmission electron microscopy (TEM), selected area electron diffraction (SAED), and scanning electron microscopy (SEM). These data allow us to relate the crystallographic internal structure of the intermediate (Figure 2) and final particles with its external shape and to determine the growth sequence of the various facets.

\section{References}

1. Huang, X. et al., Adv. Mater. 21: 4910, 2009

2. Carbó-Argibay, E. et al., Angew. Chem. Int. Ed. 49: 9397, 2010

3. Wang, Z. L. et al., Surf. Sci. 440: L809, 1999

4. Katz-Boon, H. et al., NanoLett. 11: 273, 2011

5. Carbó-Argibay, E. et al., Nanoscale, 2: 2377, 2010 

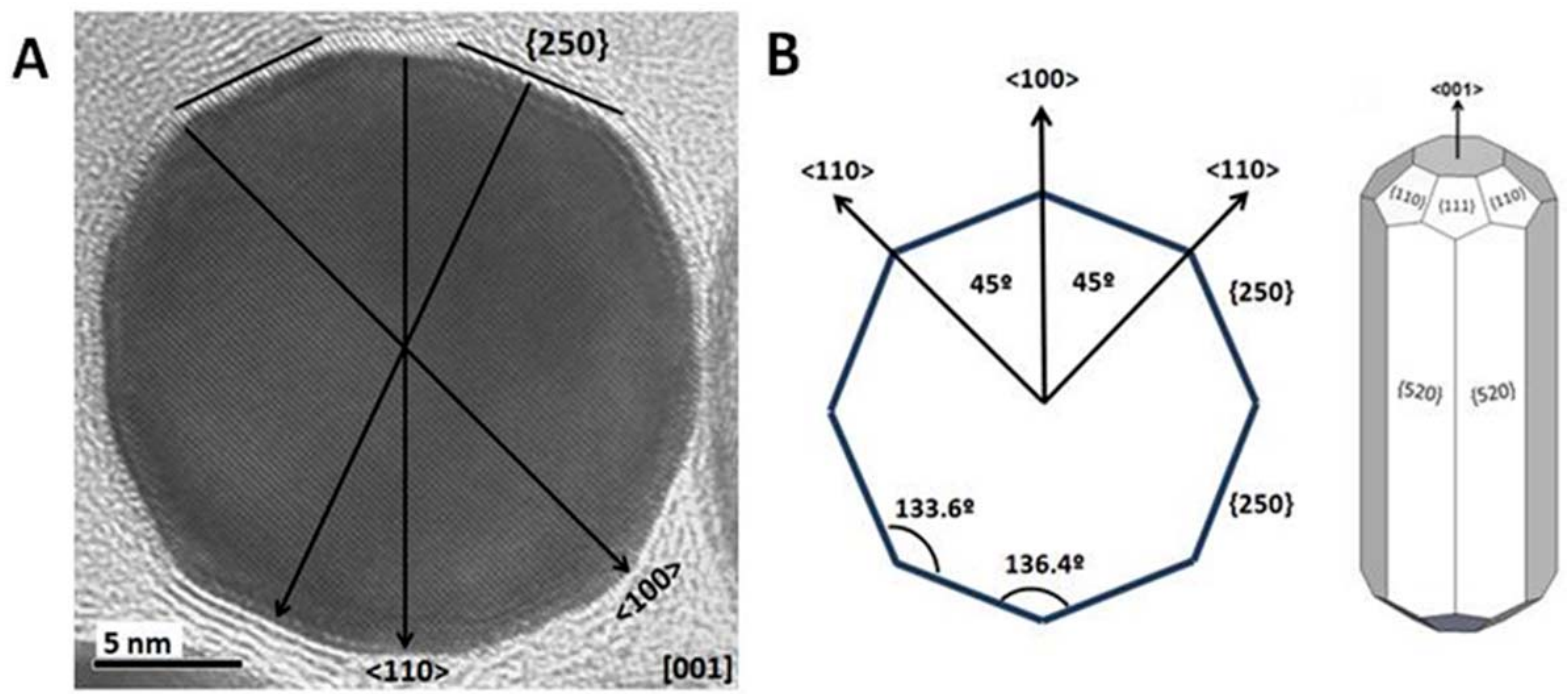

Figure 1. (A) HRTEM image of a standing gold nanorod, showing the main crystallographic directions and the indexes of the facets. (B) New proposed model for single-crystal Au Nanorods.


Figure 2. (a) TEM image of an intermediate particle oriented in the [110] zone axis (Twinning interfaces end at the middle of the edge). (b) SEM image of the same intermediate particle. 